\title{
Management of Infection Following Implant Surgery
}

\author{
SC Goel ${ }^{1 *}$, Ashank Gupta ${ }^{2}$ and Aparajita Goel ${ }^{3}$ \\ 1 Professor of Orthopaedics, India \\ 2Assistant Professor of Orthopaedics, India
}

3Assistant Professor of Pathology, India

*Corresponding author: SC Goel, Professor of Orthopaedics, India

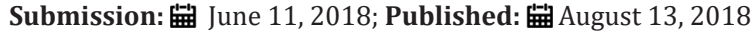

\section{Introduction}

In modern era implant surgery has become one of the commonest orthopaedic operations. Total joint replacement and fracture fixations do help by alleviating the sufferings of many patients but post-operative infection is a devastating complication. Detection of infection after implant surgery is a daunting diagnostic challenge. No test is $100 \%$ sensitive and $100 \%$ specific; thus the diagnosis of infection relies on the surgeon's judgment of the clinical presentation, and the interpretation of the results of previous investigations. The consequences of misdiagnosis are considerable.

The rate of infection after total hip arthroplasty has been relatively stable since the introduction of prophylactic antibiotics, various means of maintaining ultraclean operating rooms, and refinement of surgical technique [1]. The incidence remains approximately $1 \%$ after primary and $3 \%$ to $4 \%$ after revision hip surgery. Currently, approximately 200,000THAs per year are done in the United States, accounting for between 4,000 and 5,000 new cases per year of infected hips those require treatment. Deep sepsis represents a devastating complication for the patient and runs upto $\$ 250$ million in health care cost per year.

Staphylococcus epidermidis (31\% of cases) and S. aureus (20\%) are the most common offending organisms, whereas Streptococcus viridans (11\%), Escherichia coli (11\%), Enterococcus faecalis (8\%), and group B streptococci (5\%) are less frequently encountered. About one-third of these infections develop within 3 months, another third develop within 1 year, and the remainder develops more than 1 year after surgery. At histopathologic analysis, the inflammatory reaction accompanying the infected prosthesis is identical to that present in aseptic loosening, with one important difference: Neutrophils, usually absent in aseptic loosening, are invariably present in large numbers in infection.

\section{Clinical Presentation}

A thorough history and physical examination is of vital importance; even when a conclusive diagnosis cannot be made, a careful history and physical examination can help to guide the appropriate investigations [2]. A three-stage classification system based on the mode or timing of the presentation of infection has been described by Coventry and modified by Fitzgerald et al. [3] Type-I infections occur in the immediate postoperative period. The patient usually is seen during the first postoperative month, and the diagnosis is evident on the basis of the medical history and physical examination. Systemic signs of infection, such as fever, chills, and sweating, may be present. Pain is usually continuous. On examination, the wound may be erythematous, swollen, fluctuant and tender. Wound drainage, if present, is usually purulent.

Type-I infections are caused by infected hematomas or superficial wound infections spreading to the periprosthetic space.

Type-II infections also are believed to originate at the time of surgery, but because of a small inoculum or the low virulence of the organism, the onset of symptoms is delayed. The patient is usually seen 6 and 24 months after the procedure. The hallmark of this type of infection is a gradual deterioration in function and increase in pain. Pain is often present from the time of the original procedure; it may be activity related or it may occur at night and during rest. Often the only clue to infection is early loosening of the prosthesis. Systemic symptoms are not part of the presentation; however, there may be history of prolonged drainage at the time of the index procedure. Specific questions about a delay in the patient's discharge from the hospital, prolonged course of antibiotics, or ongoing wound drainage should be asked while the history is being obtained. The findings on examination of the hip or knee in a patient who has a type-II infection usually are nonspecific and are similar to those associated with aseptic loosening.

Type-III infections are the least common and are caused by a hematogenous spread to a previously asymptomatic hip, usually 2 years or more after the initial procedure. Generally, there is an acute febrile episode accompanied by sudden, rapid deterioration in the function of the hip or knee. The diagnosis is usually made on the basis of the history and physical examination. The patient may recall a systemic or febrile illness that was followed by symptoms in 
the hip or the knee. A Type-III infection is likely to occur in patients who are immunosuppressed, such as those who have had a renal transplant or who have been managed with immunosuppressive medications for inflammatory arthropathy or those who have recurrent episodes of bacteremia.

\section{Pathology}

Normally the host defense system eliminates the transient bacterial colonisation unless the inoculum size exceeds threshold level, host defense is impaired, tissue surfaces are traumatized, a foreign body is present or the surface or tissue is acellular. Bacterial adhesion to biomaterials leads to biomaterial centered infection and lack of successful tissue integration. Biomaterial centered infections are extraordinarily resistant to antibiotic treatment. They cannot be cured without removing the biomaterial. Foreign bodies decrease the effectiveness of host defense mechanisms and alter phenotype behavior of bacteria and susceptibility of bacteria and antibiotics.

Direct microscopy has shown bacteria to live predominantly in mixed-species biofilm community's adherent to surfaces. Bacterial cells in natural biofilm communities grow poorly (if at all) when recovered from their ecosystem and spread on agar plates, and the majority of bacterial species in natural environments cannot be cultured in any known medium. Cells within biofilms are resistant to antibiotic level 1000 times higher than those that will kill their planktonic counterpars. The chronicity of implant related infection is due to inability of antibiotics to penetrate biofilms. Even activated phagocytes cannot kill bacteria in biofilms.

Factors to be considered in musculoskeletal sepsis are adhesive bacterial colonization of a substratum, biomaterial damaged tissue, or relatively acellular tissue substratum, bacterial resistance to host defense mechanism and antibiotic therapy and type of bacteria such as staphylococcus aureus, staphylococcus epidermidis, and pseudomonas aeruginosa. Surface adhesion of bacteria or to some extent tissue cells depends on the general and relatively long range physical characteristics of the bacterium (cell), the fluid interface and the substratum. Ultimately, adhesion is based on time dependent specific protein adhesin receptor interactions, as well as carbohydrate polymer synthesis in addition to charge and physical forces.

Bacteria arrive at random near the surface of a biomaterial, foreign body, or tissue substrata by direct contamination, contiguous spreading or hematogenous seeding. After the bacteria are anchored to the substratum where nutrients are available, proliferation occurs within the polysaccharide slime and a biofilm is formed by bacterial extracapsular exopolysaccharides that bind to surfaces. After organisms adhere to biomaterials their resistance to antibiotic therapy increases and that this is specific to the type of biomaterial. This is more in polymers than metals. The killing capacity of phagocytes in the presence of a biomaterial is diminished. The foreign body surfaces trigger a slow burst and pre-empt a second burst if the phagocyte subsequently encounters an infectious organism. Bacteria can adhere to the implant surface where they are protected from antibiotics. Biofilm is formed by bacterial extracapsular exopolysaccharides that bind to surfaces. Biomaterial surfaces present binding or receptor sites for bacteria or tissue cells. There is competition between tissue and bacterial cells for the implant surface in which macrophage is the most critical host cell and cell mediated immunity is key factor. The polysaccharide film acts as a barrier to antibiotics. Antibiotics impregnated into biomaterials present a high concentration at the site of bacterial colonization.

The speed with which biofilms develop on inert surfaces or on compromised tissues (eg, dead bone) depends on the number and kind of bacterial cells, on the cleanliness of the surfaces, and the immune state of the patient. Inert materials and dead bone must be removed completely to allow the resolution of these infections, and physical removal of the causative biofilms. Surgical revisions must be covered by high doses of antibiotics, sufficient to kill the large number of planktonic bacteria that will inevitably be present in site from which large volumes of biofilm have been removed.

\section{Investigations}

To be useful, a diagnostic test must be specific as well as sensitive. A test that is sensitive but not specific can lead to multiple costly operations in many patients in whom a single intervention may have sufficed. Similarly, the specific but insensitive test will also result in additional surgical intervention because undiagnosed infection will cause any revision implant to fail. White blood count: The white count is not usually abnormal in patients having an infection following implant surgery, and it is not helpful for ruling infection out. Duncan found that the white cell count is normal in most patients who have an infection following a THA. When patient does have an abnormal count, the systemic infection is usually clinically obvious and is either type I or type III.

\section{Erythrocyte sedimentation rate}

Sedimentation Rate is a very useful screening investigation. The elevation of ESR has high sensitivity and may have a low specificity. Values of more than 30 or $35 \mathrm{~mm}$ per hour are considered to be abnormal and indicative of infection unless proven otherwise. With these values, sensitivities have been reported from 0.60 to 0.96 , and specificities from 0.65 to 1.00 . In a consecutive series of 105 revision THAs, sensitivity was $75 \%$, specificity $90 \%$. Thus, a normal ESR can safely rule out infection but an elevated ESR requires further investigation.

\section{C-reactive protein (CRP)}

C-reactive protein is an acute-phase reactant that is synthesized in the liver and is found in only trace amounts under normal condition. A CRP of more than 10mg/1 should be considered abnormal, to warrant additional investigations to rule out infection. ESR of more than $30 \mathrm{~mm} / \mathrm{hr}$ and CRP level of more than $10 \mathrm{mg} / 1$ is considered to be indicative of infection [4]. 


\section{Plain radiographs}

Plain radiography remains of limited use in the diagnosis of early infection. Most patients with infection initially have few or no radiographic changes unless they have chronic protracted infections. There are a few findings, however, that should alert the physician to the possibility of infection, especially in the absence of other causes of bony changes, such as polyethylene wear, endosteal scalloping, early loosening, rapidly progressive radiolucent lines, periosteal new bone formation, or lacy periostitis. Occasionally, plain radiographs provide clues to infection; however, they are neither sensitive nor specific.

\section{MRI}

MRI is valuable not for the diagnosis of infection, but for identification of radiolucent cement after infection is diagnosed. The cement shows up as a signal void within the femoral medullary canal. MRI can also be valuable in delineating the extent of the periprosthetic abscesses, particularly if there is an intrapelvic extension.

\section{Ultrasound}

Ultrasound also has limited role in the diagnosis of infection. It may be helpful in identifying thickened joint capsules or abscesses that could be associated with an underlying septic process. In the event of negative joint aspirate, it can also guide needle position to aspirate a surrounding abscess [5].

\section{Arthrography}

Although arthrography itself is rarely useful for the diagnosis of infection, it has recently been reported that the accumulation of dye in pockets may suggest abscess formation. As with ultrasound, arthrography may aid needle sampling of an associated extraarticular abscess when the joint aspirate is negative. Aspiration or arthrogram is probably most indicated when the ESR and CRP are elevated in a painful, symptomatic, failed THA performed initially for noninflammatory hip disease. Arthrogram allows the physician the opportunity to aspirate the joint, providing clinical specimen for aerobic and anaerobic cultures.

\section{Radionuclide imaging}

Bone scintigraphy is widely available, easily performed, and highly sensitive investigation. A study with normal results is strong evidence against a prosthetic abnormality. However, the significance of increased periprosthetic uptake is less certain. For hip prostheses, diffusely increased periprosthetic uptake is often equated with infection. The accumulation of bone-seeking tracers such as technetium- ${ }^{99 m}$ methylene diphosphonate, which localize on the surface of the bone mineral matrix, is dependent on blood flow and especially on the rate of new bone formation $[6,7]$. Consequently, any cause of accelerated new bone formation may result in increased periprosthetic activity on bone images. The diffuse pattern seen with infection is probably due to generalized osteolysis, which is also present in aseptic loosening secondary to inflammation. Therefore, these two entities may be indistinguishable at scintigraphy.

\subsection{Radiotracers used for Infection Imaging}

${ }^{99} \mathrm{mTc}$-Leucocytes, ${ }^{111} \mathrm{In}-\mathrm{WBC},{ }^{67}$ Gallium Citrate

${ }^{99} \mathrm{rnTc}$-Antigranulocyte antibodies, ${ }^{99} \mathrm{mTc}$-e- selectinantibody

${ }^{99} \mathrm{mTc}$-Interleukins ${ }^{99} \mathrm{mTc}$-Ciprofloxacin

\section{Limitations}

- $\quad$ Cost

- $\quad$ Time required for the patient to have the procedure

- Inability of the scans to yield consistently acceptable levels of sensitivity and specificity

A negative bone scan rules out infection; however, TC scan occasionally can be negative in a patient who has infection if there is an inadequate blood supply to the bone. Scans can remain positive for as long as 1 year after an uncomplicated joint replacement and for more than 2 years after insertion of prosthesis without cement. The scans cannot be used to differentiate between infection and aseptic loosening. When combined, Tc-99 and sulfur colloid 1-11 lean produce a sensitivity of $100 \%$, and specificity of $97 \%$, and accuracy to $98 \%$.

According to Larikka et al. [8], if the supplementary method were the conventional ${ }^{99 \mathrm{~m}} \mathrm{Tc}$ leukocyte imaging, 90\% accuracy would be achieved, whereas the ${ }^{99 \mathrm{~m}} \mathrm{Tc}$ ciprofloxacin imaging reaches an accuracy of $97 \%$. Use of ${ }^{99 \mathrm{~m}} \mathrm{Tc}$ leucocytes may, however, be less convenient due to time-consuming blood sampling for their in vitro labelling. Lack of ${ }^{99 \mathrm{~m}} \mathrm{Tc}$ ciprofloxacin tracer uptake in normal bone marrow, which can sometimes confuse the interpretation of the leukocyte images, should also be considered as an advantage of this procedure. In addition, ${ }^{99 \mathrm{~m}} \mathrm{Tc}$ ciprofloxacin imaging is also independent of the amount of white blood cells in circulation and thus can also be used in patients with neutropenia. On the leucocyte scan, if the abnormal uptake at the prosthesis site persists in early 30 minute image and delayed 4 hours image then it specifies infection. Furthermore, when the uptake is focal it favours infection and a diffuse marginal uptake especially at the periphery of the prosthesis favours inflammation. In this context other agents like ${ }^{99 \mathrm{~m}}$ Tc-ciprofloxin are used to differentiate infection from inflammation [9]. The functional imaging using above tracers demonstrate sensitivity and specificity of $90-95 \%$ in this clinical context.

\section{Aspiration}

Aspiration of the hip and knee is perhaps the most useful investigative tool for definitive confirmation of the presence or absence of infection. At present the role of aspiration has generated considerable debate and controversy. It has a false positive rate between 0 and $15 \%$. There is a wide variation in sensitivity and specificity as reported by various authors. One of the factors identified for this variation is that it is technique intensive. It is a 
test, which is better for ruling infection in than for ruling it out. To reduce the rate of false-negative results, all antibiotics must be discontinued for 2 or 3 weeks before the aspiration. Especially in the hip the intracapsular position of the needle should be confirmed with arthrography. Local anesthetic should be used only in the skin and not in the joint as they are bacteriostatic. If possible the specimens should be separated into three samples for culture. A diagnosis of infection is made if all three specimens are positive for the same organism and if the result coincides with the clinical profile. If only one sample is positive, the aspiration is repeated. If the result on any of the repeat aspirations is positive for the same organisms and the antibiotic-sensitive profile also is identical, then the presence of infection is confirmed.

\section{Gram stain}

The gram stain remains a very specific but a terribly insensitive test for infection; sensitivity ranges from 0 to $23 \%$. Gram stains have been associated with very high false negative rate. In less virulent infections, the bacterial load is not enough to allow reliable determination on Gram stain. In a series of 194 revision THAs and TKAs, in which intraoperative Gram stains were obtained in all patients, there were no positive Gram stains despite the presence of infection in 32 cases, as confirmed by culture.

\section{Culture}

Culture is one of the diagnostic tests in the confirmation of peri prosthetic joint infection. At least 3 tissue samples from the most inflamed areas should be sent for culture to improve yield and minimize diagnostic error. All cultures should be incubated for 5 days. Tissue culture had a sensitivity of $94 \%$, specificity of $97 \%$. Because the swab cultures are less sensitive than the tissue cultures, it is recommended sampling of inflamed tissue only during revision.

\section{Molecular analysis}

Molecular technology may be used to diagnose the presence of bacterial DNA and RNA. Polymerase chain reaction (PCR) enables the production of large amounts of specific sequences of target DNA from small quantities of starting material. This technique of determination of infection is currently under development and may hold promise for improved diagnostic accuracy. Whether PCR is too sensitive to any bacterial particles that are encountered, thereby leading to high rate of false positive results, remains to be determined.

\section{Clinical experience}

In absence of overt pus many causes of more common intraoperative scenarios of either synovitis, turbid joint fluid, fibrinous exudates, soft-tissue edema, or normal appearing tissue may often confound an accurate surgical diagnosis. Surgical experience probably has an increased role under these equivocal circumstances, and it is best to assume infection and treat accordingly. One group recently correlated the intraoperative surgical opinion with the pathologic diagnosis for a sensitivity of
$70 \%$ and a specificity of $87 \%$

1. Principles of Management

1. Antibiotic suppression

2. Open debridement

3. Closed wound treatment

4. Irrigation Arthroscopic or otherwise

5. Reaming of medullary canal

6. Implant removal

\section{Antibiotics}

Treatment of acute post operative infection is always operative and aggressive with antibiotics playing an adjuvant role. Therapy for postoperative infections should be directed toward common nosocomial pathogens, such as the staphylococci and aerobic gramnegative bacilli. The length of therapy is determined by the type of infection (cellulitis, abscess, joint infection, or osteomyelitis). Local therapy with antibiotic-impregnated polymethylmethacrylate (PMMA) beads is more helpful.

\section{Biodegradable antibiotics local delivery systems}

These vehicles are in early clinical trials, and despite the theoretical advantages, may not prove to be clinically successful. Various systems are Polylactic acid, Fibrin clots, Phlyanhydrides, Polyglycolides, Polycaprolactone and Hydroxyapatite.

\section{Debridement}

Debridement should consist of thorough removal of haematoma, infected and necrotic soft tissue (wound margins, subcutaneous and muscular tissue) and partial/ superficial closures.

\section{Irrigation with antibiotics}

Irrigation of wound with $8 \mathrm{~L}$ sterile saline, followed by $2 \mathrm{~L}$ antibiotics fluid consisting of 50,000 units of bacitracin and 1 million units of polymyxin per liter of saline is done fter putting ingress and egresstube before closing the wound. This helps in eradication of infection by removal of bacteria and providing antibiotics at site.

\section{Open wound treatment}

Open wound treatment may have to be resorted to in grossly infected wounds. Wound may be left open after debrima and covered with antiseptic dressings, tissue compatible antibiotic solution. Antibiotic impregnated beads may be used or one may employ Papineau's technique. Once wound has granulated skin cover is provided.

\section{Implant removal}

Implant removal is controversial. Some authors feel that implant providing stable fixation should be retained till union. Loose implants are removed and replaced by external fixation/ plaster/orthosis. 


\section{Infected nailing}

Infected nailings are treated by removal of implant, overreaming and exchange nailing or external fixation after thorough debridement. Reaming of medullary canal is done if infection and area of necrotic tissue is present in medullary canal and antibiotic impregnated beads or nail is used.

\section{Infected plate}

Infected plates are treated by debridement and lavage or debridement, removal of plate and external fixator or debridement and external fixator and gentamycin beads. Infection may be controlled by locally very high concentrations of antibiotics delivered "on demand" by means of a novel ultrasonic-triggered release mechanism, and biofilm bacteria may be made more susceptible to antibiotics by the imposition of weak direct-current electric fields, or by ultrasonic energy delivered at particular wavelengths [10].

\section{Conclusion}

To exclude infection as a cause of failure of an implant surgery, certain key investigations should be performed. This should help to keep the overall number of investigations to a minimum, thereby being cost effective, yet still confirming the diagnosis of infection. A diagnosis of infection is made if the clinical suspicion is high, the ESR or the CRP level, or both, are elevated for no other reason and the cultures of the aspirated fluid are positive. When in doubt we must treat as infection. Prevention is always better than cure. We must try to prevent occurrence of post operative infection with use of prophylactic antibiotics, proper preparation of patient, ultra clean air techniques, maintain strict asepsis, meticulous soft tissue handling and stable fixation.

\section{References}

1. Charnley J, Eftekhar N (1969) Postoperative infection in total prosthetic replacement arthroplasty of the hip-joint with special reference to bacterial content of the air of the operating room. Br J Surg 56(9): 641649.

2. Coventry MB (1975) Treatment of infections occurring in total hip surgery. Orthop Clin North Am 6(4): 991-1003.

3. Fitzgerald RH, Nolan DR, Ilstrup DM, Van Scoy RE, Washington JA, et al. (1977) Deep wound sepsis following total hip arthrplasty. J Bone Joint Surg Am 59(7): 847- 855.

4. Sanzen L, Carlsson AS (1989) The diagnostic value of C-reactive protein in infected total hip arthroplasties. J Bone Joint Surg Br 71(4): 638-641.

5. Graif M, Schwartz E, Strauss S, Mouallem M, Schecter M, et al. (1991) Ocult infection of hip prosthesis: Sonographic evaluation. J Am Geriatr Soc 39(3): 203-204.

6. Wegner WA, Alavi A (1991) Diagnostic imaging of musculoskeletal infection: Roentgenography; gallium, indium-labeled white blood cell, gammaglobulin.bone scintigraphy and MRI. Orthop Clin North Am 22(3): 401-418.

7. Oswald SG, Van Nostrand D, Savory CG, Callaghan JJ (1989) Three-phase bone scan and indium white blood cell scintigraphy following porous coated hip arthroplasty: A prospective study of the prosthetic tip. J Nuc Med 30(8): 1321-1331.

8. Larikka MJ, Ahonen AK, Niemelä O, Junila JA, Hämäläinen MM, et al. (2002) Comparison of ${ }^{99 \mathrm{~m} T c}$ ciprofloxacin, ${ }^{99 \mathrm{~m}} \mathrm{Tc}$ white blood cell and three-phase bone imaging in the diagnosis of hip prosthesis infections: improved diagnostic accuracy with extended imaging time. Nuclear Medicine Communications 23(7): 655-661

9. Love C, Tomas MB, Marwin SE, Pugliese PV, Palestro CJ (2001) Role of nuclear medicine in diagnosis of the infected joint replacement. Radiographics 21(5): 1229-1238.

10. Costerton JW (2005) Biofilm theory can guide the treatment of device related orthopaedic infections. Clin Orthop 437: 7-11.
Creative Commons Attribution 4.0 International License

For possible submissions Click Here
Submit Article

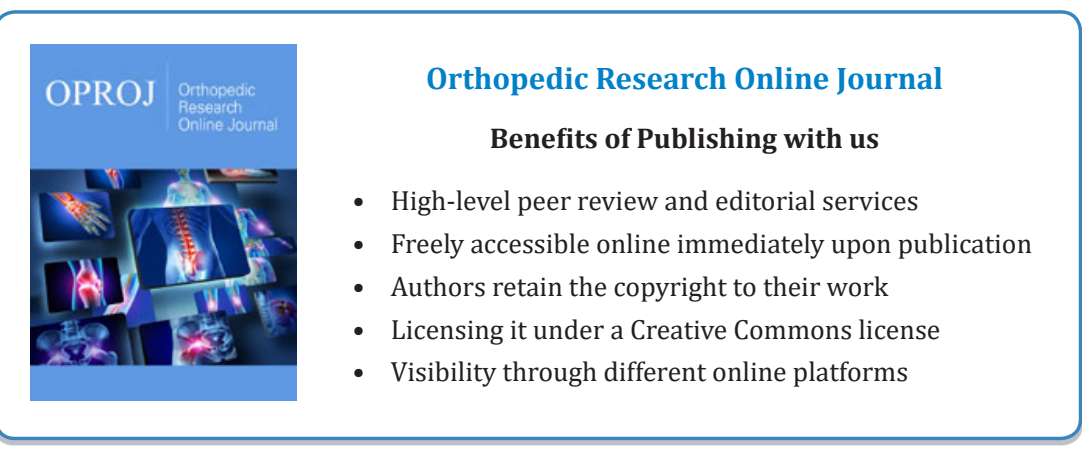

